\title{
A Comparative Study of SPM and XPM in Er-Yb Co-Doped Fiber
}

\author{
Aditya Kumar $^{1}$ [D $\cdot$ Gausia Qazi ${ }^{1}$
}

Received: 16 March 2021 / Accepted: 9 August 2021

(c) The Minerals, Metals \& Materials Society 2021

\begin{abstract}
Optical fiber communication has been the most versatile and revolutionary medium of communication that has helped connect millions of people across the globe. These traits have seen tremendous development in the sector of optical fiber communication. There are now many desired qualities expected out of an optical fiber communication such as large transmission capacity and a smaller amount of loss. Communication systems operating with higher transmission rates have found an essential role of non-linear fiber optics. The behavior of light in non-linear media is described by non-linear optics. Cross-phase modulation (XPM) and self-phase modulation (SPM) are a few non-linearities occurring in the optical fiber. Non-linear effects reduce the performance of optical fibers. In this paper, a comparative study of non-linear effects in erbium-ytterbium co-doped fiber is reviewed using simulation. The results are compared with optical systems utilizing normal fiber. The benefits of using $\mathrm{Er}-\mathrm{Yb}$ fiber have been demonstrated by analyzing effective systems comprising the Er-Yb co-doped fiber and their ability to mitigate the non-linearities.
\end{abstract}

Keywords $\mathrm{Er}-\mathrm{Yb} \cdot \mathrm{SPM} \cdot \mathrm{XPM} \cdot \mathrm{Q}$-factor

\section{Introduction}

The rapid development of erbium (Er)-doped optical fiber amplifiers (EDFA) in the $1550-\mathrm{nm}$ band has greatly improved different Er- and ytterbium ( $\mathrm{Yb}$ )-doped fiber amplifiers (EYDFAs) and 1550-nm EDFAs. ${ }^{1}$ Specifically, for high-energy applications, EYDFAs are vital, counting long-distance optical communications. ${ }^{2}$ As of late, EYDFAs have been utilized in modern specialized areas, such as ultrahigh-repetition-rate beat sources. ${ }^{3}$ EYDFs co-doped with $\mathrm{Yb}$ attain several advantages over erbium-doped fibers (EDFs) with benefits such as higher Er concentration and, significantly, a bandwidth and a greater absorption intensity around $980 \mathrm{~nm}$. High-power cladding-pumped optical fiber amplifiers utilizing multimode laser diodes use the properties of EYDFs. ${ }^{4}$ Various stages of energy levels associated with the Er-Yb system are shown through a model in Fig. 1. Yb ions

\footnotetext{
Aditya Kumar

aditya18rocks@gmail.com

Gausia Qazi

gausia.qazi@nitsri.net

1 Department of Electronics and Communication Engineering, National Institute of Technology, Srinagar, Jammu, and Kashmir, India
}

in an EYDF are excited to the ${ }^{2} \mathrm{~F}_{5 / 2}$ state by pumping light in $800-1000 \mathrm{~nm}$. Because of the energy transfer from the $\mathrm{Yb}$ ions, while they regress to the ${ }^{2} \mathrm{~F}_{7 / 2}$ state, the ${ }^{4} \mathrm{I}_{11 / 2}$ state is achieved by Er ions due to excitation. To transition to ${ }^{4} \mathrm{I}_{13 / 2}$, a non-radiative process is utilized by the $\mathrm{Er}$ ions that are excited to ${ }^{4} \mathrm{I}_{11 / 2}$, forming a population inversion between the ${ }^{4} \mathrm{I}_{13 / 2}$ and ${ }^{4} \mathrm{I}_{15 / 2}$ states. Stimulated emission causes the amplification of the incident optical signal. Yb ions and Er ions cluster together with low solubility towards a silica host and have the same ionic radius. In this manner, the separation between $\mathrm{Yb}$ ions and Er ions decreases, and energy transfer continues in an incredibly efficient way. ${ }^{5}$

There have been various works done in investigating and mitigating non-linear effects. The authors in Refs. 6, 7 and 8 have been able to reduce the non-linearities using multiple methods. The methodologies adopted by the authors in Ref. 6 use a regenerative system based on SPM with a bandwidth of $10 \mathrm{~Gb} / \mathrm{s}$; the authors in Ref. 7 have developed a regenerative system based on highly non-linear fibers (HNLF); the authors in Ref. 8 utilized a multimode non-linear fiber with a numerical approach. The work done by these authors has led us to study the benefits of using a system comprising $\mathrm{Er}-\mathrm{Yb}$ fiber to overcome the issues developed in the above positions. We have investigated the properties of Er-Yb fiber by comparing it with other similar systems consisting of 


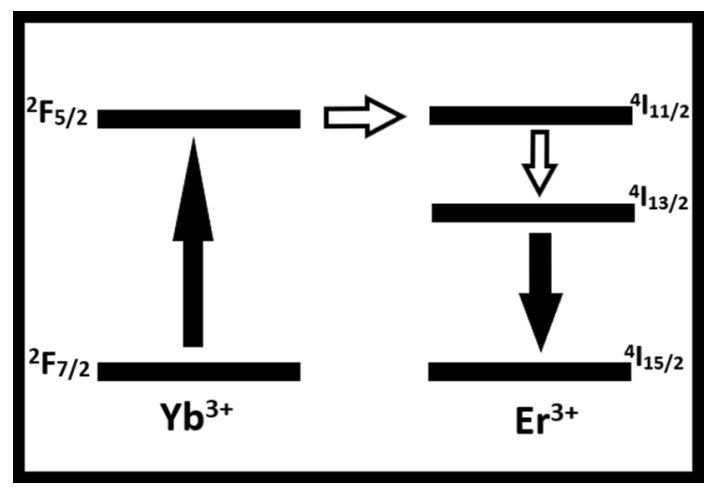

Fig. 1 Energy levels in the Er-Yb system.

only a normal fiber for transmission. The issues were poor quality factor (Q-factor) in Ref. 6 and a limited data rate. ${ }^{6-8}$

To study the benefits of using the Er-Yb fiber, we have performed a comparative study, analyzing the quality factor and data rates of an optical communication system containing Er-Yb fiber to the optical systems proposed by authors in Refs. 9 and 10. The findings observed during the comparison will help overcome the shortcomings of the above works, thus providing a simple yet more efficient system to mitigate non-linearities using Er-Yb co-doped fiber.

The paper discusses the origin of non-linearity and discusses self-phase modulation (SPM) in Sect. 2 and crossphase modulation (XPM) in Sect. 3. The simulation setup and its properties are discussed in Sect. 4. The results and analysis are presented in Sect. 5, and the conclusion is given in Sect. 6.

Non-linearity in its preliminary stage originated due to the impact of an applied field on the anharmonic motion of bounded electrons. The total polarization $(P)$ is affected by this anharmonic motion; electric dipoles induce $P$, and its value is not linear but sums up additionally as a general relation given by

$P=\varepsilon_{0} \chi^{(1)} E 1+\varepsilon_{0} \chi^{(2)} E 2+\varepsilon_{0} \chi^{(3)} E 3+\cdots$

where $\chi^{(l)}(l=1,2, \ldots)$ is $l$ th order susceptibility and permittivity of vacuum is given by $\varepsilon 0$. The linear susceptibility $\chi^{(1)}$ provides the dominant contribution to $P$. For sumfrequency generation and second harmonic generation, the second-order susceptibility $\chi^{(2)}$ is responsible. $\chi^{(2)}$ vanishes for a symmetrical molecule such as silica. Therefore, the second-order non-linear refractive effects are not exhibited by optical fibers. For non-linear effects in fibers of the lowest order, the third-order susceptibility $\chi^{(3)}$ is responsible. ${ }^{11}$ $\mathrm{Er}-\mathrm{Yb}$ fiber has been selected for analysis due to its high efficiency, low noise, ${ }^{12}$ and cost-effectiveness. ${ }^{13}$ Values of non-linearities must be kept as low as possible for effective communication. ${ }^{14}$

\section{Self-Phase Modulation}

The intensity-dependent phase transition of the fiber is triggered by the intensity-dependent refractive index. ${ }^{15}$ Kerr non-linearities for the propagating light pulse through the fiber form a separate phase transmission for the pulse height relative to the leading and trailing ends of the light pulse. Such an effect that causes modifications to the pulse spectrum is known as self-phase modulation (SPM). A timevarying frequency is created by the time-varying phase due to the instantaneous frequency of a wave, which is the time derivative of its phase. Therefore, SPM is responsible for the alteration and broadening of the frequency spectrum of the pulse. Dispersion-like effects are produced due to the spectral broadening caused by SPM, limiting the transmission rates in some of the optical communication systems of the long-haul category, depending on its chromatic dispersion and type of fiber utilized for transmission. ${ }^{16} \mathrm{~A}$ time-varying refractive index is caused due to the increase in intensity variation. It falls as the pulse goes past at any single point in the fiber, while the optical pulse propagates through the fiber. A non-linear phase shift, which varies with time, is caused due to the temporally varying index change. The optical field phase changes are given by Ref. 17

$\phi=\left(n+n_{2}|E|^{2}\right) k_{02} L$

The linear refractive index is denoted by $n$, and $n_{2}$ indicates the non-linear refractive index of the fiber. $\left(|E|^{2}\right.$ is the optical signal intensity). The free-space wave number is denoted by $\mathrm{k} 0$ and contains the value of $2 \pi / \lambda, \lambda$ is the optical signal wavelength, and $L$ denotes the fiber length. ${ }^{17}$ Intensity dependence of phase fluctuations causes different phase shifts in different parts of the pulse leading to frequency chirping. A frequency shift in the upper side is produced in the leading edge of the optical pulse, whereas the frequency shift in the lower side is produced in the trailing edge. Therefore, a significant effect of SPM is to broaden the pulse spectrum, with the temporal shape untouched. In high-transmitted power systems, SPM effects are more pronounced because the effect of chirping is proportional to signal power transmitted. ${ }^{18}$

An induced phase shift in SPM is proportional to the intensity, occurring due to the non-linear refractive index value. ${ }^{19}$ The formula between refractive index and intensity can be described as:

$\Delta n=n_{2} I(t)$

where the non-linear index is denoted by $n_{2}$ and optical intensity is denoted by $I(t)$. The pulse phase is given by: 
$\phi(t)=\omega_{0} t-k x=\omega_{0} t-\frac{2 \pi}{\lambda_{0}} n(I) L$

here $L$ is the propagated distance by the pulse, and the carrier frequency is given by $\omega_{0}$ is the and $\lambda_{0}$ denotes the wavelength of the pulse. ${ }^{19}$

\section{Cross-Phase Modulation}

Effects of XPM and SPM are related. Distinguishable and overlapping pulses with different wavelengths or polarizations are involved in this case. Phase modulation of the overlapping pulse(s) is caused by the intensity variations of one pulse that modulates the fiber's refractive index. This modulation of phase in SPM converts into a modulation in frequency that broadens the spectrum of the pulse. XPM is, therefore, displayed as a mechanism of crosstalk between channels. This occurs when the dispersive optical fiber undergoes intensity modulation during transmission, or when phase encoding is used with the increase in the number of channels, the strength of XPM increases. Decreasing the channel spacing also increases the strength of XPM.

Nevertheless, there is no exchange of energy among channels, differentiating the effect from other crosstalk processes in which a signal power increase in one channel arises only through a power reduction in another. It can be inferred that the overall intensity is the square of the number of two electric field amplitudes, which makes the total strength of XPM twice that of SPM. This influence is diminished as pulses with different wavelengths or polarizations are typically not balanced by group velocity, and thus overlap is not preserved. ${ }^{16}$ The shift for the $i$ th channel in an $N$-channel transmission system can be given as, ${ }^{18}$

$\phi_{n l}^{i}=\left(P_{i}+2 \sum_{n \neq i}^{N} p i\right) k_{n l} L_{\mathrm{eff}}$

The above equation contains a factor 2 , which has its origin in the form of non-linear susceptibility and indicates the same amount of power; the XPM is twice as effective as SPM. The first term and the second term in the above equation represent the contribution of SPM and XPM, respectively. It can be inferred that XPM is effective only when the interacting signals are superimposed in time. ${ }^{17}$ Total non-linear phase shift in a fiber of length $L:{ }^{19}$

$\phi_{N L}=\left(\frac{2 \pi}{\lambda}\right) n_{2}\left(\left|I_{1}\right|^{2}+b\left|I_{2}\right|^{2}\right)$

\section{Simulation Setup of the System}

OptiSystem $09^{20}$ simulation software platform was used to study the various parameters responsible for surging SPM and XPM effects. ${ }^{9}$ The authors in Ref. 10 have also utilized OptiSystem to study non-linearities in fiber. A system to study SPM analysis has been created and is shown in Fig. 2 . The system operates at a wavelength of $1550 \mathrm{~nm}$. The power provided to the input is varied from $10 \mathrm{dBm}$ to $20 \mathrm{dBm}$. The length of fiber is assumed to be $100 \mathrm{~km}$. The transmission rate is fixed at $10 \mathrm{~Gb} / \mathrm{s}$. The Er-Yb fiber length is set at $1 \mathrm{~m}$, and a pump laser is used to amplify the signal and improve efficiency. SPM effect is introduced in the fiber. The analysis to find the eye-diagram is done using a bit error rate (BER)

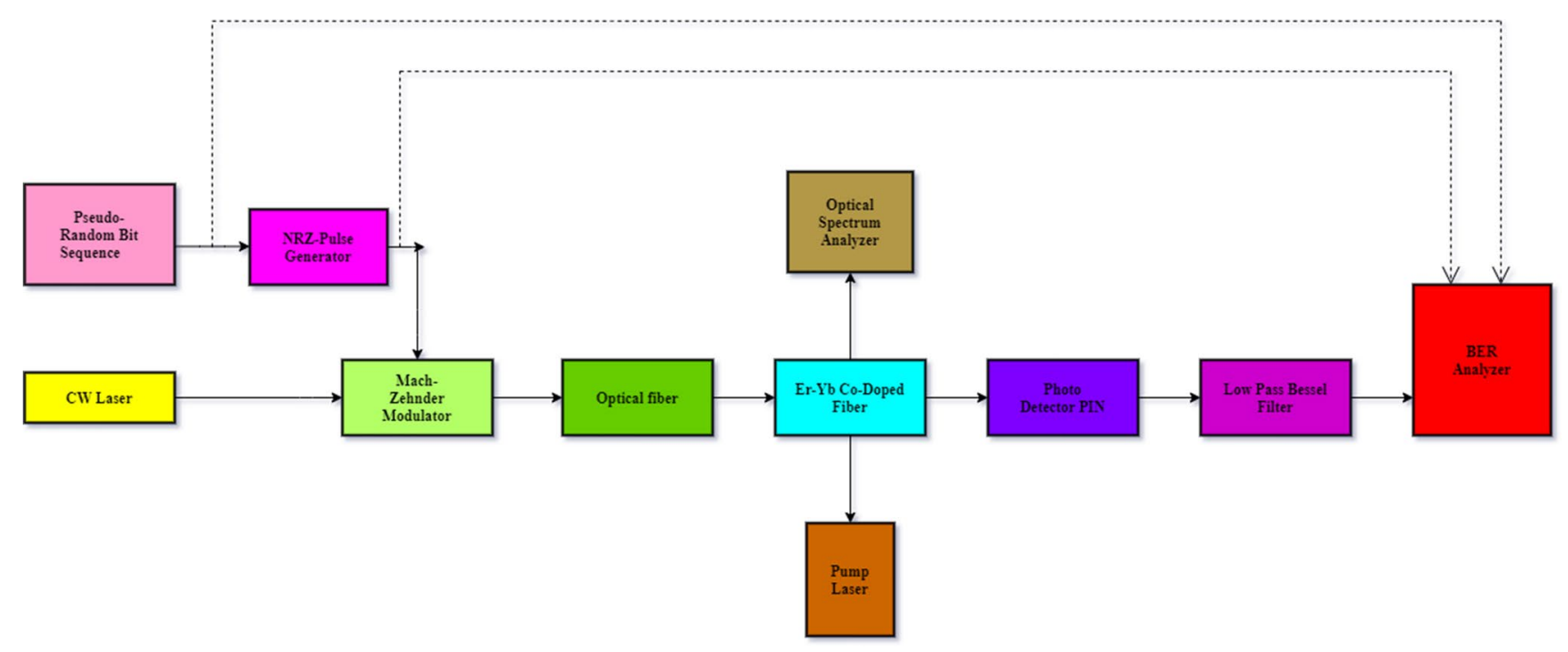

Fig. 2 SPM simulation setup. 
analyzer to devise the Q-factor that determines the distortion in the signal. A photodetector PIN, low-pass Bessel filter (LPBF) with a cut-off frequency set at $0.65 *$ bitrate, and a BER analyzer constitute the receiver section (Table I).

A wavelength divison multiplexing (WDM) system consisting of two channels has been utilized to analyze the effects of XPM, and it is shown in Fig. 3. To generate signals in the system, two optical Gaussian pulse generators and two user-defined bit sequence generators are used to provide input signals. Multiplexing of two signals over a single channel is done using an ideal multiplexer. The signal after the process of multiplexing is further passed to the fiber and passed through EYDF. The length of the EYDF is set at $1 \mathrm{~m}$. The length of optical fiber is assumed to be $50 \mathrm{~km}$. A BER analyzer is used to obtain the Q-Factor for the assumed signal. A photodetector PIN, LPBF with a cut-off frequency set at $0.70 *$ (bitrate), BER analyzer, and $3 \mathrm{R}$ regenerator constitute the receiver section (Table II).

Table I Important parameters and their values pertaining to the SPM system

\begin{tabular}{ll}
\hline Parameter & Value \\
\hline Bitrate & $10 \times 10^{9} \mathrm{Bits} / \mathrm{s}$ \\
Length of optical fiber & $100 \mathrm{~km}$ \\
Length of Er-Yb Co-doped fiber & $1 \mathrm{~m}$ \\
Core-radius of Er-Yb Co-doped fiber & $2 \mu \mathrm{m}$ \\
Doping-radius of Er-Yb Co-doped fiber & $2 \mu \mathrm{m}$ \\
Er-ion density of Er-Yb Co-doped fiber & $51.4 \times 10^{24} \mathrm{~m}^{-3}$ \\
Yb-ion density of Er-Yb Co-doped fiber & $620 \times 10^{24} \mathrm{~m}^{-3}$ \\
Er metastable lifetime & $10 \mathrm{~ms}$ \\
Yb metastable lifetime & $1.5 \mathrm{~ms}$ \\
Low-pass Bessel filter cut-off-frequency & $0.65 \times$ bitrate \\
\hline
\end{tabular}

\section{Results and Analysis}

In this section, the analysis of the effects of SPM and XPM on the fiber is shown. Input power and transmission rates are some parameters that have been changed over time to provide conclusive evidence of the effects on the fiber.

\section{Eye-Diagram Analysis of Self-Phase Modulation}

Marvin and Pratheesh ${ }^{9}$ demonstrated a simulation based on optical fiber to analyze the SPM effect. They obtained various values of the Q-factor by varying the input power of the source. They varied the power from 10 to $20 \mathrm{dBm}$ and found that the Q-factor completely vanished at $20 \mathrm{dBm}$ power input. ${ }^{9}$

We analyzed the Q-Factor by varying the power from 10 $\mathrm{dBm}$ to $20 \mathrm{dBm}$ and obtained the following results. Initially, the input power is set at $10 \mathrm{dBm}$ and $15 \mathrm{dBm}$.

The output optical signals associated are shown in Fig. 4a and $b$, respectively.

Now, we vary the input power from $17 \mathrm{dBm}$ to $20 \mathrm{dBm}$. The output optical signals are shown in Fig. 5a and 5b, respectively.

We observed that the Q-factor at $10 \mathrm{dBm}$ power input was 5.06 , and at $15 \mathrm{dBm}$, the power input was 8.38 . When

Table II Important parameters and their values in the XPM system

\begin{tabular}{ll}
\hline Parameter & Value \\
\hline Length of optical fiber & $50 \mathrm{~km}$ \\
Length of Er-Yb Co-doped fiber & $1 \mathrm{~m}$ \\
Core-radius of Er-Yb Co-doped fiber & $2 \mu \mathrm{m}$ \\
Doping-radius of Er-Yb Co-doped fiber & $2 \mu \mathrm{m}$ \\
Er-ion density of Er-Yb Co-doped fiber & $51.4 \times 10^{24} \mathrm{~m}^{-3}$ \\
Yb-ion density of Er-Yb Co-doped fiber & $620 \times 10^{24} \mathrm{~m}^{-3}$ \\
Er metastable lifetime & $10 \mathrm{~ms}$ \\
Yb metastable lifetime & $1.5 \mathrm{~ms}$ \\
Low-pass Bessel filter cut-off-frequency & $0.70 \times$ bitrate \\
\hline
\end{tabular}

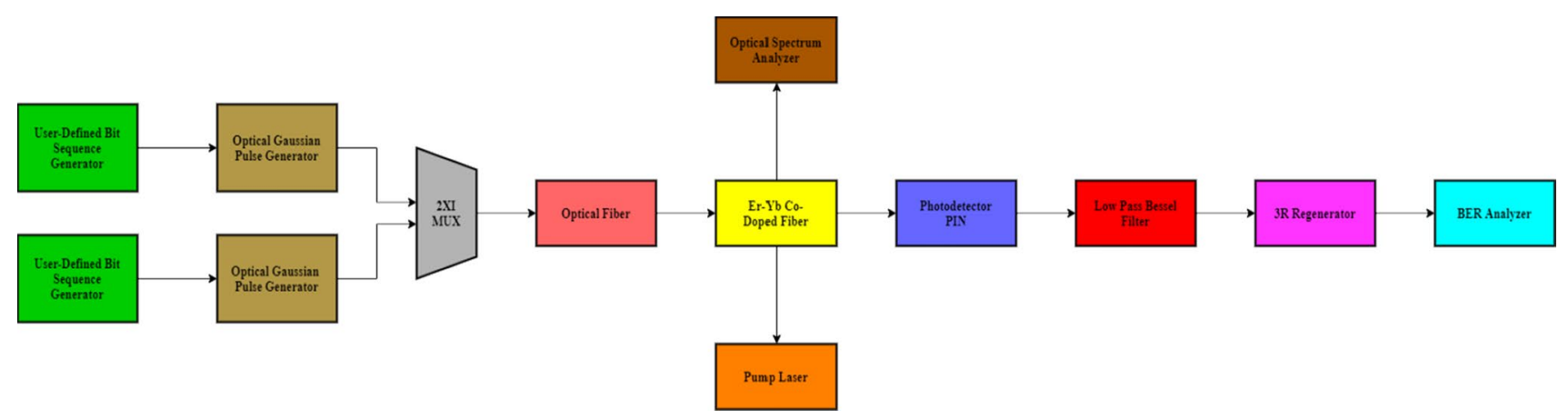

Fig. 3 XPM simulation setup. 


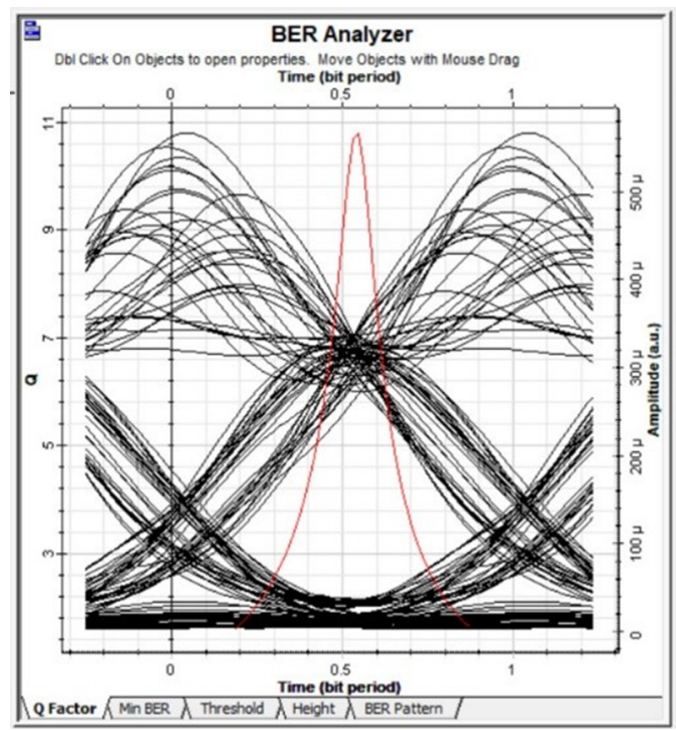

(a)

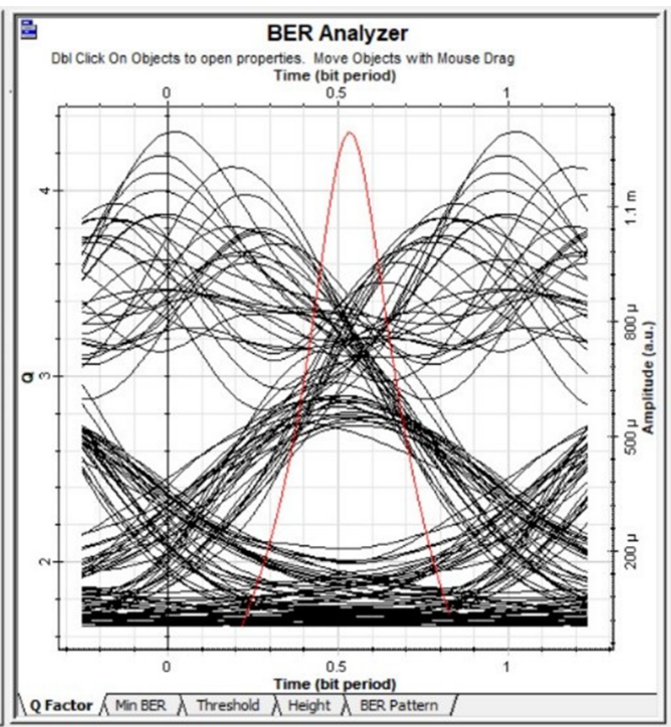

(b)

Fig. 4 (a) Eye-diagram for SPM at $10 \mathrm{dBm}$ input, (b) Eye-diagram for SPM at $5 \mathrm{dBm}$ input.

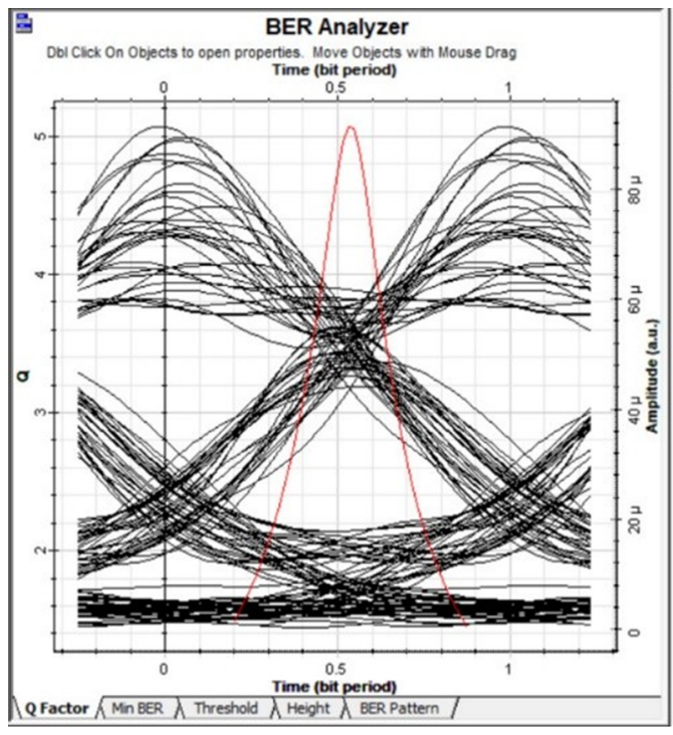

(a)

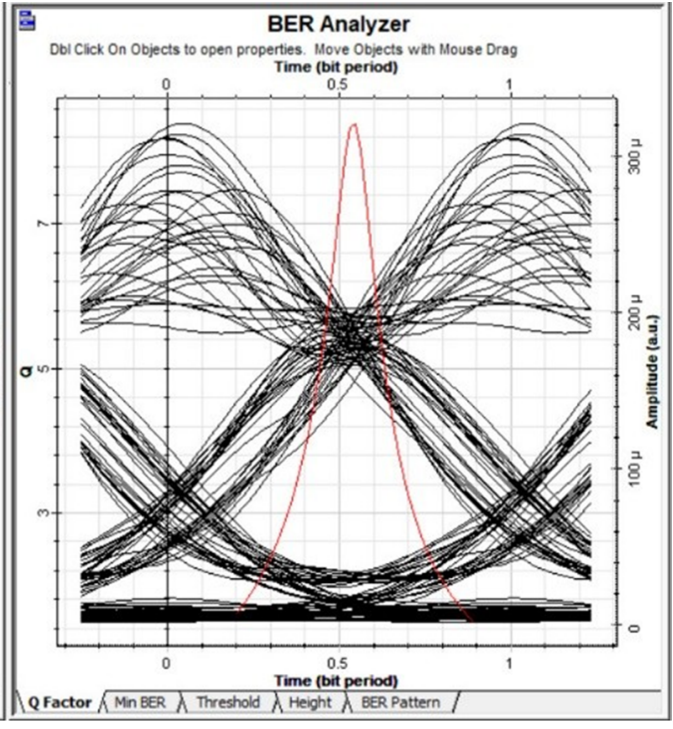

(b)

Fig. 5 (a) Eye-diagram for SPM at $17 \mathrm{dBm}$ input, (b) Eye-diagram for SPM at $20 \mathrm{dBm}$ input.

Table III Eye-diagram parametric values (SPM).

\begin{tabular}{lllll}
\hline Parameter & $10 \mathrm{dBm}$ & $15 \mathrm{dBm}$ & $17 \mathrm{dBm}$ & $20 \mathrm{dBm}$ \\
\hline Max. Q-factor & 5.06 & 8.38 & 10.79 & 4.32 \\
Min. BER & $2.02815 \times 10^{-7}$ & $2.4637 \times 10^{-17}$ & $1.64342 \times 10^{-27}$ & $7.45267 \times 10^{-6}$ \\
Eye height & $1.79711 \times 10^{-5}$ & $107 \times 10^{-6}$ & $214 \times 10^{-6}$ & $196 \times 10^{-6}$ \\
Threshold & $3.08781 \times 10^{-5}$ & $9.16885 \times 10^{-5}$ & $118 \times 10^{-6}$ & $291 \times 10^{-6}$ \\
Decision inst. & 0.53 & 0.55 & 0.55 & 0.53 \\
\hline
\end{tabular}


the power input was increased to $17 \mathrm{dBm}$, the Q-factor increased to 10.79 , and at $20 \mathrm{dBm}$, the Q-factor decreased to 4.31. The Er-Yb fiber is able to compensate SPM at lower power inputs. Table III gives the values of parameters related to the eye-diagram. At higher power input rates, the eye-opening decreases, thus SPM grows and depletes the signal. The Er-Yb fiber gave a Q-Factor of 4.31 at 20 $\mathrm{dBm}$ input, whereas the Q-factor in the normal fiber vanished. ${ }^{9}$ The comparative results have been demonstrated in Fig. 6 indicating possible transmission quality in the case of $\mathrm{Er}-\mathrm{Yb}$ fiber. The Er-Yb system generates a better Q-Factor

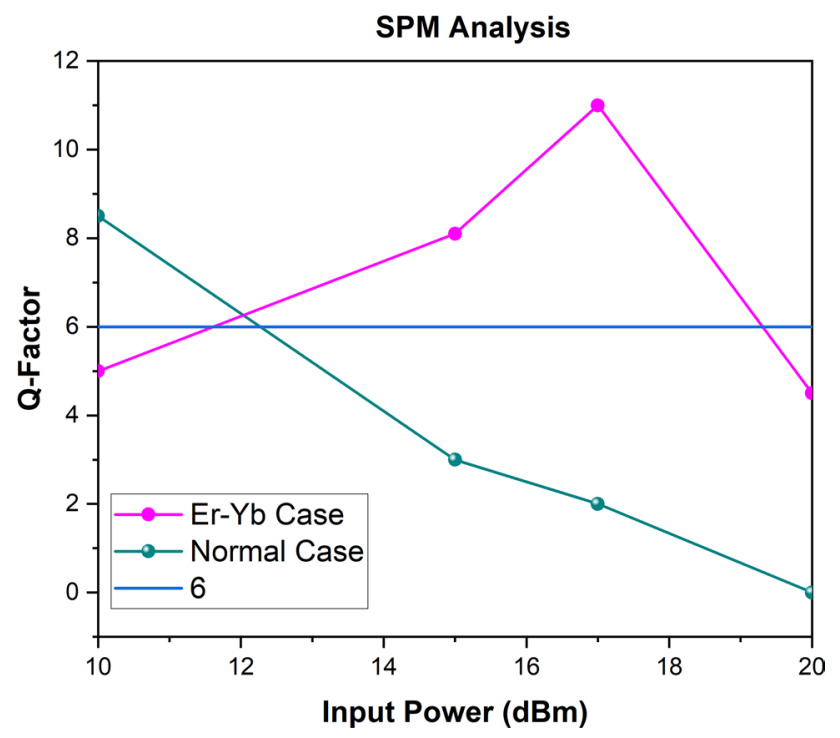

for higher input power conditions, whereas in Ref. 9, the Q-Factor deteriorates and vanishes. As a point of benefit, the system with Er-Yb at $20 \mathrm{dBm}$ input power manages to provide transmission quality, but the other system completely fails. Thus, Er-Yb fiber has better operating conditions.

\section{XPM Analysis by Eye-Diagram}

\section{Based on Varying Transmission Rates}

In this section for the simulation, the rate of transmission is altered from $10 \mathrm{~Gb} / \mathrm{s}$ to $40 \mathrm{~Gb} / \mathrm{s}$ to analyze the effect of XPM on varying transmission rates. The $\mathrm{Q}$-factor is considered to determine the strength of the signal.

Initially, the input transmission rate is set at $10 \mathrm{~Gb} / \mathrm{s}$ and $20 \mathrm{~Gb} / \mathrm{s}$. The corresponding output eye-diagrams are portrayed in Fig. 7a and b, respectively.

Now, the input transmission rate to the fiber is varied from $30 \mathrm{~Gb} / \mathrm{s}$ to $40 \mathrm{~Gb} / \mathrm{s}$. The corresponding eye-diagrams are portrayed in Fig. 8a and b, respectively. The parametric values of the eye-diagram are shown in Table IV.

The Q-factor obtained at 10, 20, 30, and $40 \mathrm{~Gb} / \mathrm{s}$ are 18.78, 5.16, 3.7, and 5.4. These data are shown in Fig. 9, indicating a decrease in Q-Factor. Still, the rate of decrease is slower in this fiber, increasing slightly at the end, evidently showing that the Er-Yb fiber supports higher transmission rates. Thus, Er-Yb fiber can give good quality factors at various transmission rates and has a better-compensating ability than normal fiber. ${ }^{10}$

Fig. 6 Comparative study of SPM.

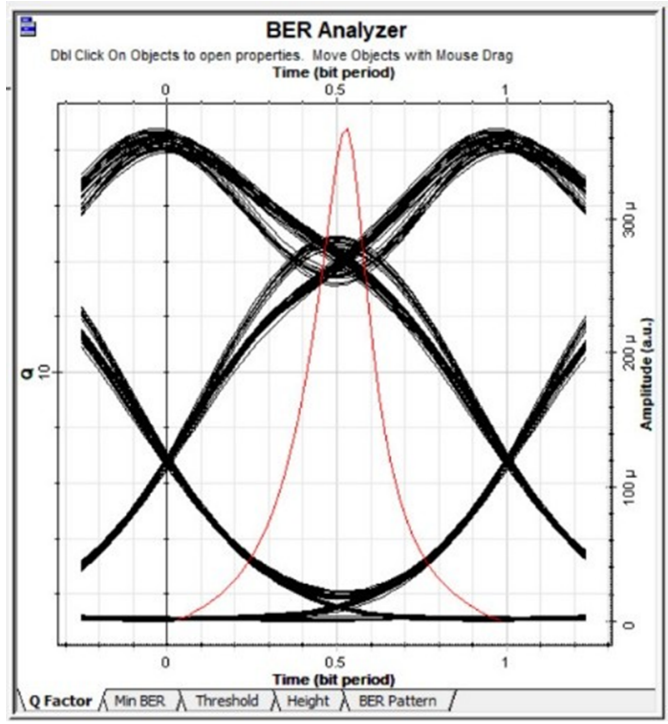

(a)

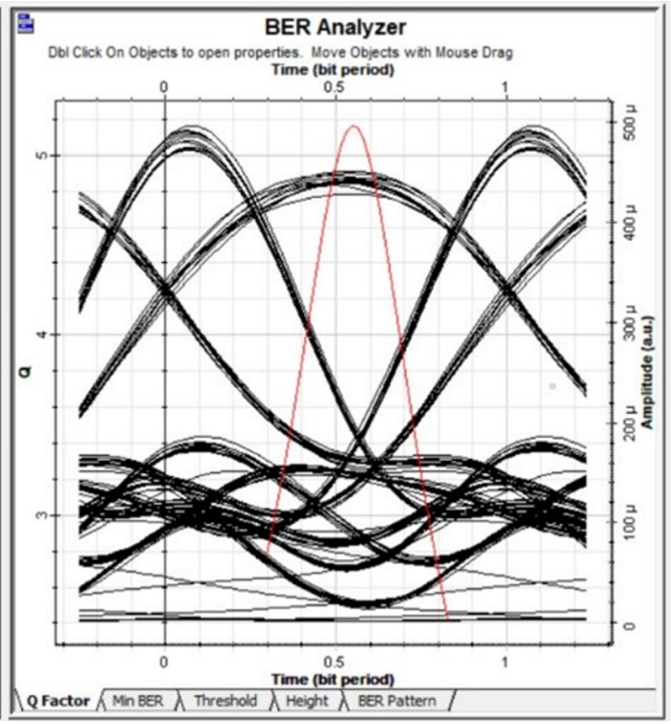

(b)

Fig. 7 (a) Eye-diagram for XPM at $10 \mathrm{~Gb} / \mathrm{s}$ input, (b) Eye-diagram for XPM at $20 \mathrm{~Gb} / \mathrm{s}$ input. 


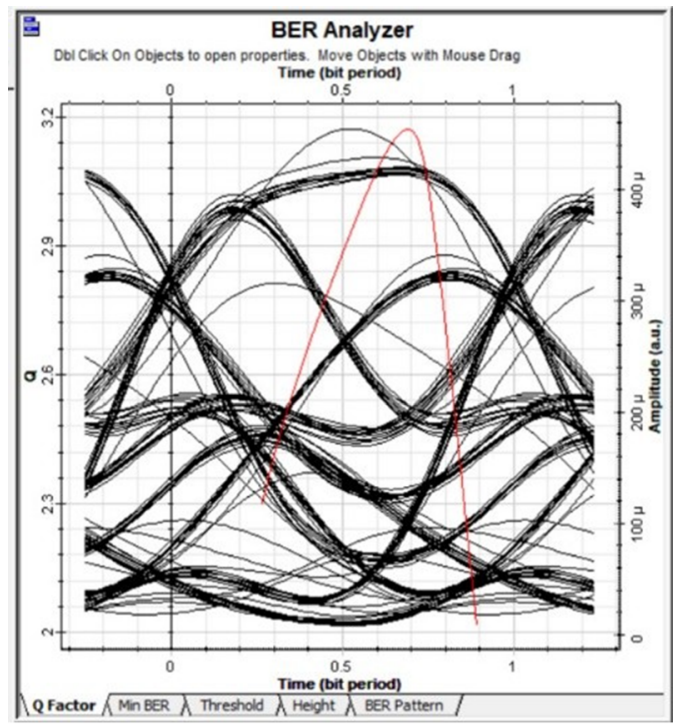

(a)

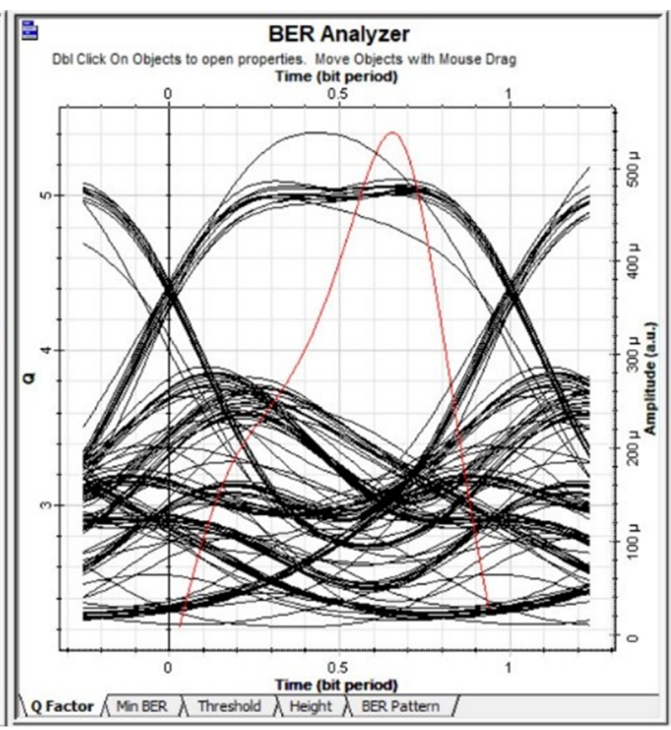

(b)

Fig. 8 (a) Eye-diagram for XPM at $30 \mathrm{~Gb} / \mathrm{s}$ input, (b) Eye-diagram for XPM at $40 \mathrm{~Gb} / \mathrm{s}$ input.

Table IV Eye-diagram parametric values (XPM).

\begin{tabular}{lllll}
\hline Parameter & $10 \mathrm{~Gb} / \mathrm{s}$ & $20 \mathrm{~Gb} / \mathrm{s}$ & $30 \mathrm{~Gb} / \mathrm{s}$ & $40 \mathrm{~Gb} / \mathrm{s}$ \\
\hline Max. Q-factor & 18.79 & 5.17 & 3.18 & 5.4 \\
Min. BER & $4.94205 \times 10^{-79}$ & $1.22285 \times 10^{-17}$ & $752 \times 10^{-6}$ & $2.95298 \times 10^{-8}$ \\
Eye height & $216 \times 10^{-6}$ & $139 \times 10^{-6}$ & $1.59369 \times 10^{-5}$ & $160 \times 10^{-6}$ \\
Threshold & $125 \times 10^{-6}$ & $411 \times 10^{-5}$ & $402 \times 10^{-6}$ & $400 \times 10^{-6}$ \\
Decision inst. & 0.53 & 0.55 & 0.68 & 0.66 \\
\hline
\end{tabular}

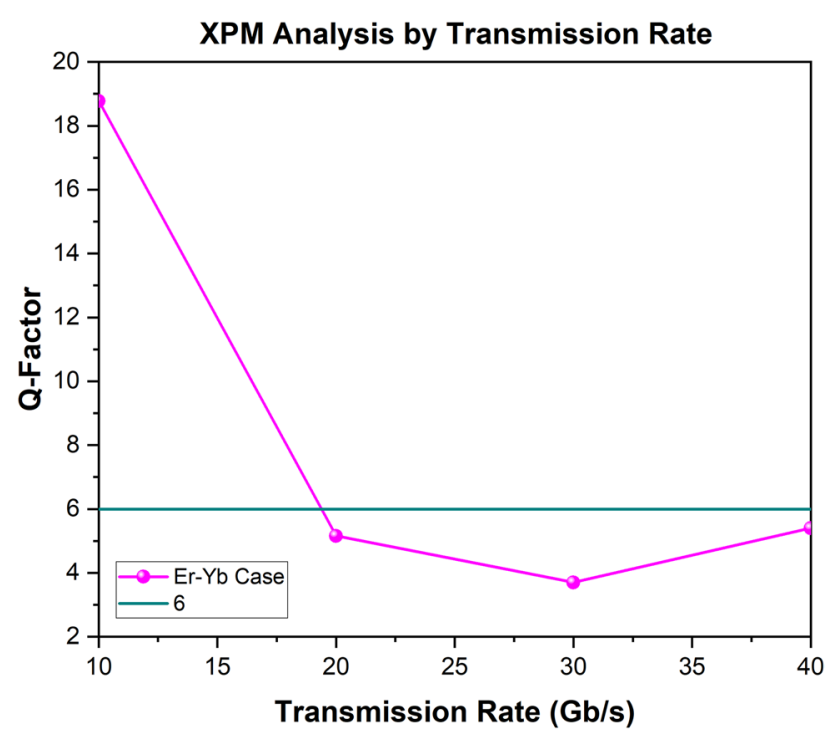

Fig. 9 Study of XPM by transmission rates.

\section{Based on Varying Input Power}

Bhusari, Deshmukh, and Jagdale ${ }^{10}$ demonstrated a simulation based on optic fiber to analyze XPM effects and have obtained various values of $\mathrm{Q}$-factor by varying the input power of the source from $0 \mathrm{dBm}$ to $12 \mathrm{dBm}$. They found that the Q-factor decreased from 4.7 to 2.7 , causing degradation of the input signal. ${ }^{10}$

We analyzed the Q-Factor by varying powers from $0 \mathrm{dBm}$ to $20 \mathrm{dBm}$ and obtained the following results. Initially, the input power is set at $0 \mathrm{dBm}$ and $5 \mathrm{dBm}$.

The output optical signals associated are shown in Fig. 10a and b, respectively.

Now, the power given to the input of the fiber is varied from $10 \mathrm{dBm}$ to $20 \mathrm{dBm}$. The corresponding eye-diagrams are shown in Fig. 11a and b, respectively.

Here, despite varying power inputs, the Q-factor constantly stays around 5 and decreases slightly at the end. Figure 12 indicates the comparative plot between the considered 


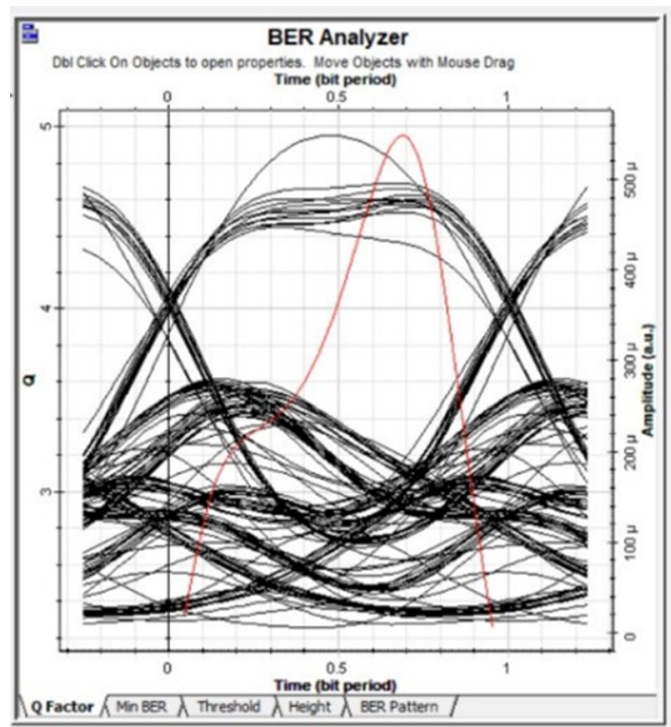

(a)

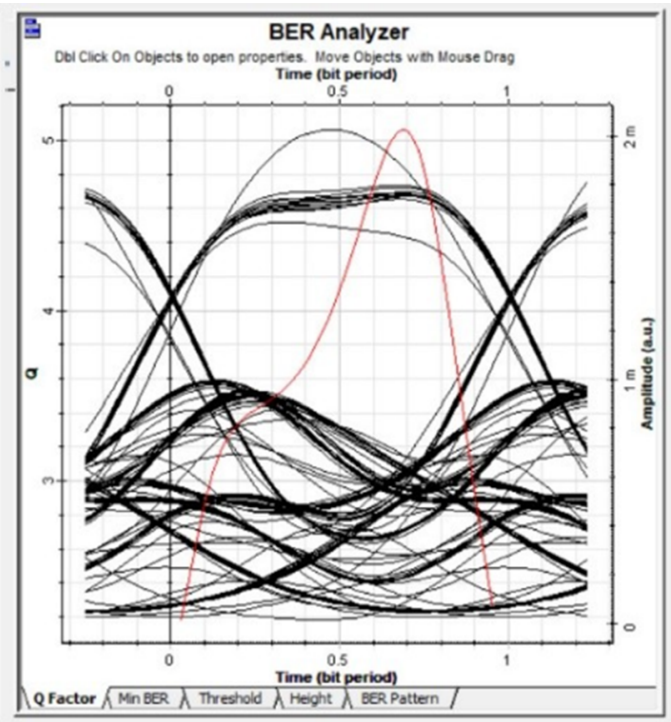

(b)

Fig. 10 (a) Eye-diagram for XPM at $0 \mathrm{dBm}$ input, (b) Eye-diagram for XPM at $5 \mathrm{dBm}$ input.

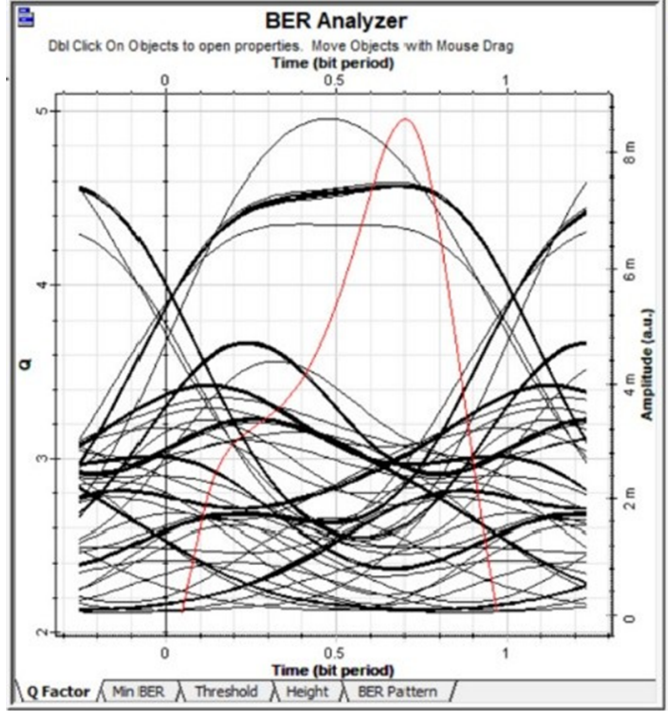

(a)

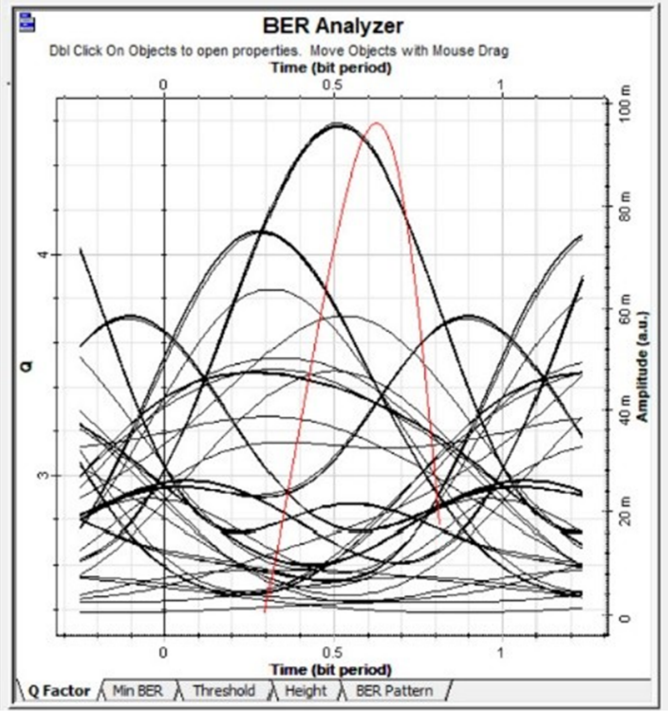

(b)

Fig. 11 (a) Eye-diagram for XPM at $10 \mathrm{dBm}$ input, (b) Eye-diagram for XPM at $20 \mathrm{dBm}$ input.

systems. The Er-Yb fiber generates higher quality signals with better Q-Factor than the system in Ref. 10. Thus, Er-Yb fiber can give better results as compared to normal fiber utilized in Ref. 10. The parametric values of the eye-diagram are shown in Table IV (Table V).

\section{Conclusion}

Mitigation of non-linearities has been effectively shown to be achieved with optical communication systems comprising Er-Yb fiber, generating good Q-Factor, and providing a 


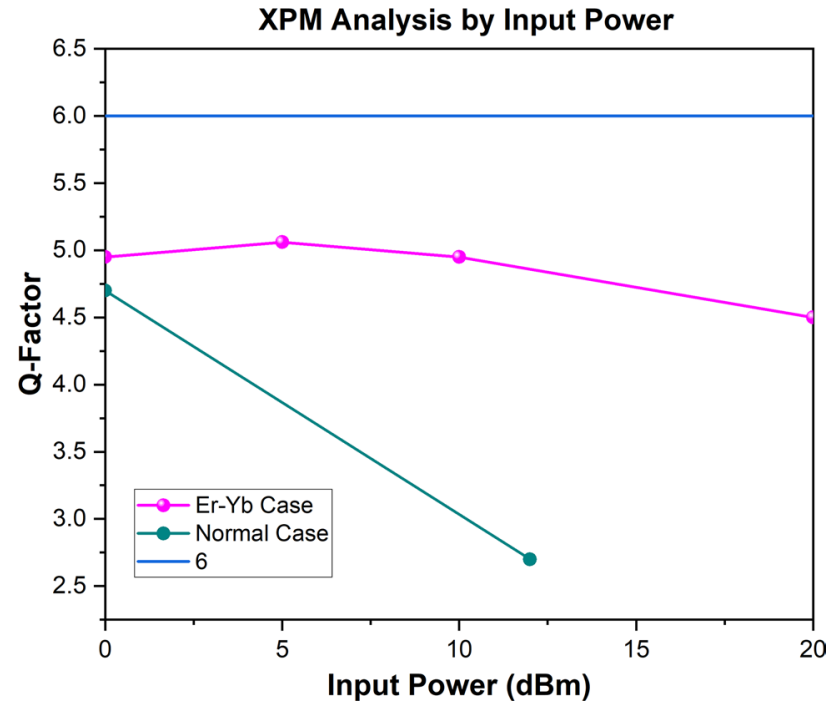

Fig. 12 Comparative study of XPM by input power.

Table V Eye-diagram parametric values (XPM).

\begin{tabular}{|c|c|c|c|c|}
\hline Parameter & $0 \mathrm{dBm}$ & $5 \mathrm{dBm}$ & $10 \mathrm{dBm}$ & $20 \mathrm{dBm}$ \\
\hline $\begin{array}{l}\text { Max. } \\
\text { Q-factor }\end{array}$ & 4.95 & 5.06 & 4.95 & 4.5 \\
\hline Min. BER & $\begin{array}{c}3.28823 \times \\
10^{-79}\end{array}$ & $\begin{array}{r}1.91865 \\
\times 10^{-17}\end{array}$ & $\begin{array}{c}3.37875 \times \\
10^{-7}\end{array}$ & $\begin{array}{c}2.10913 \\
10^{-6}\end{array}$ \\
\hline Eye height & $140 \times 10^{-6}$ & $543 \times 10^{-6}$ & $222 \times 10^{-6}$ & $236 \times 10^{-6}$ \\
\hline Threshold & $387 \times 10^{-6}$ & $1458 \times 10^{-5}$ & $6287 \times 10^{-6}$ & $8594 \times 10^{-5}$ \\
\hline $\begin{array}{l}\text { Decision } \\
\text { inst. }\end{array}$ & 0.68 & 0.68 & 0.70 & 0.62 \\
\hline
\end{tabular}

higher signal quality. Non-linear effects in Er-Yb co-doped fiber are relatively lower than the normal fiber, and it can mitigate the non-linearities to a better extent. Systems capacity and signal quality are reduced by SPM and XPM affect. SPM grows and drains the signal at a comparatively lower rate when input power is increased. XPM also performs a similar slower degradation of signal. Thus, Er-Yb co-doped fiber may be adopted if the signal quality required is of eminent importance. The study can be further extended by analyzing the effects of different input signal waveforms on the fiber and studying the nature of four-wave mixing.
Conflict of interest The authors declare that they have no known competing financial interests or personal relationships that could have appeared to influence the work reported in this paper.

\section{References}

1. J.E. Townsend, W.L. Barnes, and S.G. Crubb, Electron. Lett. 27, 1958 (1991).

2. S. Gupta, D. Engin, D. Pachowicz, J.-L. Fouron, J. Lander, X. Dang, S. Litvinovitch, T. Chuang, K. Puffenberger, F. Kimpel, R. Utano, and M. Wright, in Proc. SPIE 9739, Free Space Laser Communication and Atmospheric Propagation XXVIII, 97390V (2016)

3. Wu. Zhaohui, S. Yang, H. Chen, M. Chen, and S. Xie, IEEE Photon. Technol. Lett. 29, 409 (2017).

4. G. Canat, J.-C. Mollier, J.-P. Bouzinac, G.M. Williams, B. Cole, L. Goldberg, Y. Jaouën, and G. Kulcsar, J. Opt. Soc. Am. B 22, 2308 (2005).

5. P. Ribeiro, D.L. Andrews, and M. Raposo eds., Optics, Photonics and Laser Technology. (Springer International Publishing, Springer Nature Switzerland, 2017).

6. S. S. M. Yaacob, N. S. M. Shah, N. N. Shamsuddin, B. Das, ARPN J. Eng. Appl. Sci. 10(19) (2015)

7. F. Wen, S. Sygletos, C. P. Tsekrekos, X. Zhou, Y. Geng, B. Wu, $\mathrm{K}$. Qiu, and S. K. Turitsyn, in 19th international conference on transparent optical networks (ICTON), 1 (2017)

8. L.G. Wright, Z.M. Ziegler, P.M. Lushnikov, Z. Zhu, M.A. Eftekhar, D.N. Christodoulides, and F.W. Wise, IEEE J. Sel. Top. Quantum Electron. 24, 1 (2016).

9. M. S. Koshy, P. Pratheesh, Int. J. Eng. Res. Technol 3(03) (2014)

10. N.B. Shraddha, U.D. Vikas, and S.J. Shantanu, Int. J. Sci. Technol. Eng. 2, 136 (2016).

11. G.P. Agrawal, Nonlinear Fiber Optics, 3rd ed., (San Diego, CA: Academic Press, 2001).

12. J.T. Kringlebotn, J.L. Archambault, L. Reekie, J.E. Townsend, G.G. Vienne, and D.N. Payne, Electron. Lett. 30, 972 (1994).

13. Z.J. Chen, J.D. Minelly, and Y. Gu, Electron. Lett. 32, 1812 (1996).

14. V. Singh, S. Kumar, and P.K. Dimri, Optik 186, 177 (2019).

15. A.K. Vyas, Optik 168, 184 (2018).

16. J.M. Senior, Optical Fiber Communications: Principles and Practice, 3rd ed., (Pearson Education, 2014).

17. S. P. Singh, and N. Singh, Progr. Electromagn. Res., PIER73, 249 (2007)

18. N. Kikuchi, and S. Sasaki, J. Lightwave Technol. 13, 868 (1995).

19. I. Chatti, F. Baklouti, F. Chekir, and R. Attia, in 2019 International Conference on Smart Applications, Communications and Networking, Sharm El Sheik, Egypt, 1 (2019)

20. www.optiwave.com (Manual can be found online)

Publisher's Note Springer Nature remains neutral with regard to jurisdictional claims in published maps and institutional affiliations. 